Original Article

\title{
The influence of trunk muscle strength on walking velocity in elderly people with sarcopenia
}

\author{
Kota Kato, RPT, MS ${ }^{1,2)}$, Yasuhiko Hatanaka, RPT, PhD ${ }^{2)}$ \\ 1) Shutaikai Hospital: 8-1 Shirokita-cho, Yokkaichi-city, Mie 510-0823, Japan \\ 2) Suzuka University of Medical Science, Japan
}

\begin{abstract}
Purpose] Sarcopenia increases the risk of falls and fractures. However, its relationship with walking, which is the generation mechanism of falls, has not been clarified. The purpose of this study was to clarify the trunk muscle strength and the characteristics of walking, in elderly people with sarcopenia. [Participants and Methods] The participants were 40 elderly people aged 65 years and over. The participants were able to walk without assistance and were attending outpatient rehabilitation or community day-care centers. The assessment and measurement items included: the presence or absence of sarcopenia (using the diagnostic criteria of the Asian Working Group for Sarcopenia), lower limb and trunk muscle strength, and characteristics of walking. The participants were divided into two groups depending on the presence or absence of sarcopenia, and a comparison was made between the two groups. [Results] The participants in the sarcopenia group had significantly lower trunk extension muscle strength as compared to the participants in the non-sarcopenia group. In addition, the hip joint maximum flexion moment, ankle joint maximum plantar flexion moment, and walking velocity of participants in the sarcopenia group were significantly lower than those in the non-sarcopenia group. [Conclusion] This study revealed that weakness of the trunk muscle strength causes a decrease in walking velocity in elderly people with sarcopenia. Key words: Sarcopenia, Trunk muscle strength, Walking velocity
\end{abstract}

(This article was submitted Oct. 14, 2019, and was accepted Nov. 28, 2019)

\section{INTRODUCTION}

According to the Annual Report on the Aging Society ${ }^{1)}$, the percentage of elderly people aged 65 and over in the total population of the world (aging rate) rose from $5.1 \%$ in 1950 to $8.3 \%$ in 2015 . Furthermore, it is expected to rise to $17.8 \%$ by 2060 , and aging is expected to rapidly progress in the next half century. With the increase of the aging population, it is important to prevent and improve the weakness experienced by elderly people.

Sarcopenia is the "age-related decrease of muscle mass" proposed by Rosenberg in 19892). In 2010, the European Working Group on Sarcopenia in Older People (EWGSOP) ${ }^{3}$ ) recommended that sarcopenia be diagnosed based on both loss of muscle mass and loss of muscle function (loss in physical ability or muscle weakness). In 2014, the Asian Working Group for Sarcopenia (AWGS) ${ }^{4}$ published diagnostic criteria of sarcopenia for Asians. In addition, sarcopenia was adopted in the International Classification of Diseases (ICD-10) in 2016, and is now attracting attention as a "disease" worldwide. The prevalence of sarcopenia is reported to be approximately 5 to $20 \%$ among community-dwelling elderly people aged 65 and older $^{5-7)}$. Sarcopenia leads to movement disorders and reduced ability to perform activities of daily living, increasing the risk of falls and fractures ${ }^{7,8)}$.

When an elderly person suffers a fall it is liable to result in injury such as a fracture, and this acts as a trigger to subsequent deteriorating health ${ }^{9}$. In the United States of America, 20-30\% of older people who fall suffer moderate to severe injuries such as bruises, hip fractures, or head trauma ${ }^{10)}$. In addition, medical care and nursing care costs for falls in Japan exceed 900

*Corresponding author. Kota Kato (E-mail: kota.kato26@gmail.com)

(C2020 The Society of Physical Therapy Science. Published by IPEC Inc.

(c) (1) $\odot$ This is an open-access article distributed under the terms of the Creative Commons Attribution Non-Commercial No DerivaCC BY NC ND tives (by-nc-nd) License. (CC-BY-NC-ND 4.0: https://creativecommons.org/licenses/by-nc-nd/4.0/) 
billion yen annually ${ }^{11)}$. Thus, in the elderly countries such as Japan, preventing falls in the elderly is a very important issue. Falls by elderly people occur in a variety of scenarios, but it is reported that approximately $60 \%$ of them occur during walking $^{12)}$. However, it is not yet clear whether there is a specific relationship between sarcopenia, which is the cause of falls, and walking. Therefore, in the past, we have investigated the relationship between sarcopenia and walking in elderly people ${ }^{13)}$. The results showed that, in the sarcopenia group, despite no clear difference in lower limb muscle strength compared to the control group, a decrease in walking velocity was observed. This implied that the cause of the decrease in walking velocity in sarcopenia may be not only reduced lower limb muscle strength but also other factors. We hypothesized that one of the other factors might be trunk muscles.

There are two main functions of the trunk muscle. The first is the movement of the trunk, which is mainly performed by surface muscles such as the rectus abdominis muscles and the erector spinae muscles. The second is to maintain posture, which is mainly performed by deep muscles such as the transverse abdominal muscles and multifidus muscles, and has an important role in fixing the trunk during walking. Therefore, it is said that post-trunk changes and back pain are more likely to occur when trunk muscle strength decreases, which affects walking ability ${ }^{14-16)}$. The causes of the decrease in trunk muscle strength include aging, disease, and disuse due to inactivity, and in recent years, research has been conducted on the relationship between sarcopenia and trunk muscle strength ${ }^{17)}$. In addition, changes in muscle mass in trunk muscles do not decrease as rapidly as those in limbs, but have been reported to decrease with age in both men and women ${ }^{18,19)}$. For this reason, we thought elderly people with sarcopenia have an unstable posture such as a kyphosis due to a decrease in trunk muscle strength, and it is difficult to fix the trunk during walking, so that the walking velocity may have decrease. However, to the best of our knowledge, there are few reports on sarcopenia and trunk muscles, and a detailed research is not sufficient. Therefore, this study aimed to investigate the relationship between sarcopenia and trunk muscles in elderly people and to clarify the characteristics of walking in sarcopenia.

\section{PARTICIPANTS AND METHODS}

The participants were 40 elderly people (14 males and 26 females, $83.1 \pm 5.7$ years) aged 65 years and over who could walk with no assistance and who were attending outpatient rehabilitation or community day-care centers. People who were unable to undergo bioelectrical impedance analysis due to pacemakers or other medical electronic devices, those who had a history of cerebrovascular disorder, those who were undergoing injury treatment, and those who had a Mini-Mental State Examination (MMSE) score of 23 or less were excluded from the study. This study was conducted with the approval of the medical corporation Shutaikai ethics committee (Authorization number: 2017-014), written informed consent was obtained and the rights of the participants were protected.

The history of falls over the past year was ascertained by interview. In addition, a fall was defined as "unintentionally coming to the ground or some lower level other than as a consequence of sustaining a violent blow, loss of consciousness, or sudden onset of paralysis as in a stroke or an epileptic seizure"20).

The presence or absence of sarcopenia was determined using the diagnostic criteria of the AWGS ${ }^{4}$. For measurement of muscular mass (skeletal muscle mass), a body composition meter (InBody 230, InBody Japan Inc., Tokyo, Japan) was used. Then, the value $\left(\mathrm{kg} / \mathrm{m}^{2}\right)$ obtained by dividing the limb muscle mass $(\mathrm{kg})$ obtained by Bioelectrical Impedance Analysis (BIA) by the square of the height $(\mathrm{m})$ was used for evaluation. Measurement of muscular strength (grip strength) was performed using a grip strength meter (TTM Smedlay's Dynamo Meter $100 \mathrm{~kg}$ Yo II, Tsutsumi Seisakusho Co., Ltd., Tokyo, Japan), measuring twice each on the left and right side, with the largest value recorded. Measurement of physical function (10 m usual walking velocity) was performed using a stopwatch, measurement was performed twice, and the average value was recorded.

The measurement of lower limb muscle strength used a muscle strength meter ( $\mu$ Tas F-1, ANIMA Inc., Tokyo, Japan) with reference to previous studies ${ }^{21-24}$. Hip joint flexion muscle strength, hip joint extension muscle strength, knee joint extension muscle strength, and ankle joint plantar flexion muscle strength, which are the major muscular strengths affecting walking, were measured. Measurement of each muscular strength was performed during maximum isometric contraction of about 3 seconds. Measurements were carried out twice on each side at intervals of 30 seconds or more. Then, each maximum value was adopted, and the value obtained by dividing the left and right average value (kgf) by the body weight (kg) was expressed as the muscle force value $(\mathrm{kgf} / \mathrm{kg})$. For each measurement, the procedure was fully explained to the participants, the participants practiced beforehand, and performed the test, and when the posture was lost, the measurement was performed again. In addition, the inter-rater and intra-rater reliability of this measurement has been confirmed to be an intraclass correlation coefficients of 0.9 or higher in previous studies ${ }^{21-24)}$ and prior surveys.

The measurement of trunk muscle strength used a muscle strength meter ( $\mu$ Tas F-1, ANIMA Inc., Tokyo, Japan) and a back muscle strength meter (T.K.K.5402, Takei Scientific Instruments Co., Ltd., Niigata, Japan) with reference to previous studies $^{25,26)}$. Trunk flexion muscle strength and trunk extension muscle strength were measured. Measurement of each muscular strength was performed during maximum isometric contraction of about 3 seconds. The measurement was performed twice at intervals of 30 seconds or more. Then, the maximum value (kgf) was adopted, and the value divided by the body weight $(\mathrm{kg})$ was taken as the muscle force value $(\mathrm{kgf} / \mathrm{kg})$. For each measurement, the procedure was fully explained to the participants, the participants practiced beforehand, and performed the test, and when the posture was lost, the measurement 
was performed again. In addition, the inter-rater and intra-rater reliability of this measurement has been confirmed to be an intraclass correlation coefficients of 0.9 or higher in prior surveys.

To measure the characteristics of walking, one force plate (Accu Gait, AMTI Inc., Watertown, MA, USA) and four digital high vision video cameras (GZ-G5-B, JVC KENWOOD Inc., Kanagawa, Japan) were used. We installed digital high vision video cameras around the $4 \mathrm{~m}$ walking path where the force plate was installed, and photographed walking motion from front to back and from left and right. The sampling frequency was set to $60 \mathrm{~Hz}$, and an optical-synchronization signal generator (PH-145, DKH Inc., Tokyo, Japan) was used for synchronization. The walking velocity was taken as the comfortable walking velocity of each participant and the number of times measurement was performed was set to three on each side. Markers (diameter $15 \mathrm{~mm}$ ) were applied at 10 points: on both sides of each of the acromion, the greater trochanter, the lateral joint space, the lateral malleolus, and the head of the fifth metatarsal bone. The captured motion picture was imported into a 3D motion analysis system (Frame-DIAS V, DKH Inc., Tokyo, Japan), and the 3D spatial coordinates of each marker were calculated. In addition, the data obtained from the 3D spatial coordinates and the force plate were converted into DIFF (Date Interface File Format) and then converted into Excel format using DIFF gait and Wave Eyes, which are software provided by the clinical gait analysis group ${ }^{27}$. The joint angles and joint moments of the hip joint, the knee joint and the ankle joint, and the inclination angle of the trunk during one gait cycle were calculated from the respective data in Excel format.

For evaluation of cognitive function, the Mini-Mental State Examination (MMSE) was used.

General information (gender, age, history of present illness, past medical history) on each participant was collected from their medical records.

The participants were divided into a sarcopenia group and a non-sarcopenia group according to the presence or absence of sarcopenia. Attributes (gender, age, prevalence of musculoskeletal diseases) and physical characteristics (height, weight) of the participants, and each evaluation and measurement item were compared between the two groups. Fisher's direct stochastic method, the $\chi^{2}$ independence test, and Mann-Whitney U test were used for comparison between the two groups. JSTAT statistical analysis software was used for statistical analysis, and the significance level was set at $\mathrm{p}<0.05$.

\section{RESULTS}

Of the 40 participants, there were 19 people ( 2 males and 17 females, average age $85.5 \pm 4.2$ years) who were judged as having sarcopenia (sarcopenia group) and 21 people (12 males and 9 females, average age $80.9 \pm 6.1$ years) who were judged as not having sarcopenia (non-sarcopenia group). The prevalence of sarcopenia was $47.5 \%$ (Table 1 ).

Table 2 shows the results of comparison of the participants' attributes and physical characteristics between the sarcopenia group and the non-sarcopenia group. Between the two groups, regarding the gender ratio, the proportion of women (89.5\%) was significantly higher in the sarcopenia group while that of men (57.1\%) was significantly higher in the non-sarcopenia group $(\mathrm{p}<0.01)$. As for age, the mean age in the sarcopenia group was $85.5 \pm 4.2$ years, and in the non-sarcopenia group was $80.9 \pm 6.1$ years, thus the sarcopenia group was significantly older than the non-sarcopenia group $(\mathrm{p}<0.05)$. As for prevalence

Table 1. Attributes (gender, age) of the participants and prevalence of sarcopenia

\begin{tabular}{lc}
\hline Participants (n) & 40 (Males: 14, Females: 26) \\
Age (years) & $83.1 \pm 5.7$ \\
Age group (n (\%)) & \\
$65-69$ & $1(2.5)$ \\
$70-79$ & $8(20.0)$ \\
$80-89$ & $27(67.5)$ \\
90 and over & $4(10.0)$ \\
Sarcopenia group (n (\%)) & $19(47.5)$ \\
Non-Sarcopenia group (n (\%)) & $21(52.5)$ \\
\hline
\end{tabular}

Table 2. Comparison of attributes and physical characteristics of participants between the sarcopenia group and the nonsarcopenia group

\begin{tabular}{lcc}
\hline & $\begin{array}{c}\text { Sarcopenia group } \\
\mathrm{n}=19\end{array}$ & $\begin{array}{c}\text { Non-Sarcopenia } \\
\text { group } \\
\mathrm{n}=21\end{array}$ \\
\hline Gender (n (\%)) & $2(10.5)$ & $12(57.1)$ \\
Male & $17(89.5)$ & $9(42.9) * *$ \\
Female & $85.5 \pm 4.2$ & $80.9 \pm 6.1 *$ \\
Age (years) & $11(57.9)$ & $6(28.6)$ \\
Prevalence of musculoskeletal diseases (n (\%)) & \\
Existing fracture & $6(31.6)$ & $4(19.0)$ \\
Knee osteoarthritis & $0(0)$ & $1(4.8)$ \\
Hip osteoarthritis & $9(47.4)$ & $3(14.3) *$ \\
Osteoporosis & $4(21.1)$ & $5(23.8)$ \\
Spinal canal stenosis & $4(21.1)$ & $1(4.8)$ \\
Spondylosis deformans & $1(5.3)$ & $0(0)$ \\
Rheumatoid arthritis & $1.47 \pm 0.07$ & $1.57 \pm 0.09^{* *}$ \\
Height $(\mathrm{m})$ & $44.6 \pm 6.4$ & $58.3 \pm 8.8^{* *}$ \\
Weight $(\mathrm{kg})$ & & \\
\hline *p $<0.05, * * \mathrm{p}<0.01$. & &
\end{tabular}


of musculoskeletal diseases, the prevalence of osteoporosis in the sarcopenia group was $47.4 \%$, and in the non-sarcopenia group was $14.3 \%$, thus the sarcopenia group was significantly higher than the non-sarcopenia group ( $<<0.05$ ). As for physical characteristics, height and body weight were significantly lower in the sarcopenia group than in the non-sarcopenia group $(\mathrm{p}<0.01)$.

Table 3 shows the results of comparison of each evaluation and measurement item between the sarcopenia group and the non-sarcopenia group. Among the two groups, there was no significant difference in fall rates over the past year, but the rate in the sarcopenia group was $31.6 \%$, while that in the non-sarcopenia group was $19.0 \%$, with the sarcopenia group showing a tendency to be higher. As for lower limb and trunk muscle strength, there was no significant difference in lower limb muscle strength. However, in trunk muscle strength, the trunk extension muscle strength was $0.46 \pm 0.30 \mathrm{kgf} / \mathrm{kg}$ for the sarcopenia group and $0.76 \pm 0.30 \mathrm{kgf} / \mathrm{kg}$ for the non-sarcopenia group, showing a significant lowering in the sarcopenia group compared to the non-sarcopenia group $(\mathrm{p}<0.01)$. In each joint angle and joint moment during one walking cycle, in terms of joint angles, the hip joint extension angle at the terminal stance $(\mathrm{p}<0.05)$ and the ankle plantar flexion angle at the pre-swing $(\mathrm{p}<0.01)$ was significantly lower in the sarcopenia group than in the non-sarcopenia group. In addition, knee joint flexion angle at the terminal stance $(p<0.05)$, knee joint flexion angle at the terminal swing $(p<0.05)$, ankle joint dorsal flexion angle at the terminal stance $(p<0.01)$, and trunk anteversion angle $(p<0.05)$ were significantly higher in the sarcopenia group than in the non-sarcopenia group. In terms of joint moments, hip joint maximum flexion moment at the terminal stance $(p<0.01)$, knee joint extension moment at the pre-swing $(p<0.05)$, and ankle joint maximum plantar flexion moment at the terminal stance $(p<0.05)$ were significantly lower in the sarcopenia group than in the non-sarcopenia group (Fig. 1). As for walking parameters, walking velocity $(p<0.01)$, stride length $(p<0.05)$, and walking rate $(p<0.05)$ were all significantly lower in the sarcopenia group than in the non-sarcopenia group.

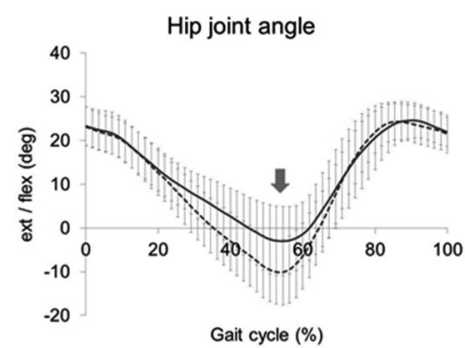

Hip joint moment
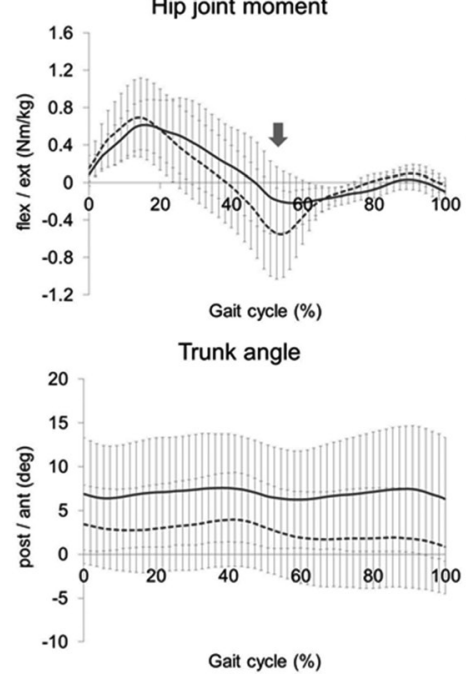

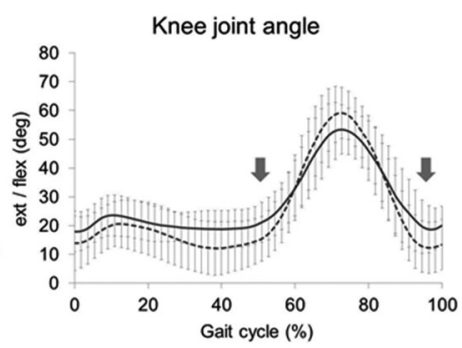

Knee joint moment

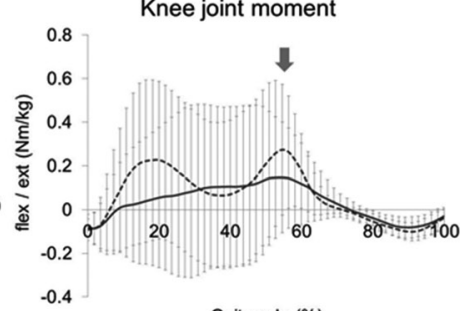

Gait cycle (\%)

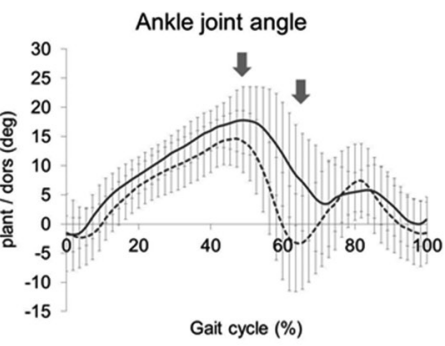

Ankle joint moment

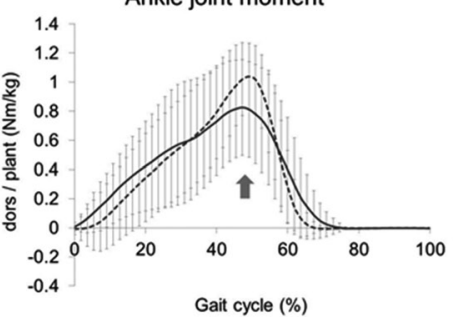

Gait cycle (\%)

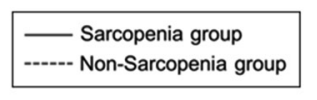

Fig. 1. The joint angles and joint moments of the hip joint, the knee joint and the ankle joint, and the inclination angle of the trunk during one gait cycle. Percentile values for the sarcopenia group are presented as a solid line, and for the non-sarcopenia group as a dotted line. The positions where significant differences were observed between the sarcopenia group and the non-sarcopenia group are indicated by arrows $(\Rightarrow)$. 
Table 3. Comparison of each evaluation and measurement item between the two groups: the sarcopenia group and the non-sarcopenia group

\begin{tabular}{|c|c|c|}
\hline & $\begin{array}{c}\text { Sarcopenia group } \\
\mathrm{n}=19\end{array}$ & $\begin{array}{l}\text { Non-Sarcopenia group } \\
n=21\end{array}$ \\
\hline \multicolumn{3}{|l|}{ History of falls of the past year (n (\%)) } \\
\hline History of falls & $6(31.6)$ & $4(19.0)$ \\
\hline No history of falls & $13(68.4)$ & $17(81.0)$ \\
\hline \multicolumn{3}{|l|}{ Isometric lower limb muscle strength (kgf/kg) } \\
\hline Hip joint flexion & $0.20 \pm 0.05$ & $0.24 \pm 0.09$ \\
\hline Hip joint extension & $0.24 \pm 0.09$ & $0.30 \pm 0.15$ \\
\hline Knee joint extension & $0.31 \pm 0.07$ & $0.37 \pm 0.13$ \\
\hline Ankle joint plantar flexion & $0.41 \pm 0.12$ & $0.49 \pm 0.18$ \\
\hline \multicolumn{3}{|l|}{ Isometric trunk muscle strength $(\mathrm{kgf} / \mathrm{kg})$} \\
\hline Trunk flexion & $0.10 \pm 0.05$ & $0.11 \pm 0.05$ \\
\hline Trunk extension & $0.46 \pm 0.30$ & $0.76 \pm 0.30^{* *}$ \\
\hline \multicolumn{3}{|l|}{ Joint angle (deg) } \\
\hline \multicolumn{3}{|l|}{ Hip joint } \\
\hline Flexion [Initial contact] & $23.4 \pm 4.4$ & $23.0 \pm 4.4$ \\
\hline Extension [Terminal stance] & $5.1 \pm 7.3$ & $11.0 \pm 7.3^{*}$ \\
\hline Flexion [Terminal swing] & $25.4 \pm 4.1$ & $24.9 \pm 4.3$ \\
\hline \multicolumn{3}{|l|}{ Knee joint } \\
\hline Flexion [Initial contact] & $17.5 \pm 6.8$ & $13.5 \pm 9.2$ \\
\hline Flexion [Loading response] & $24.3 \pm 6.8$ & $21.5 \pm 8.2$ \\
\hline Flexion [Terminal stance] & $17.6 \pm 6.3$ & $11.1 \pm 8.8^{*}$ \\
\hline Flexion [Initial swing] & $58.0 \pm 5.4$ & $60.9 \pm 7.9$ \\
\hline Flexion [Terminal swing] & $17.7 \pm 7.4$ & $11.2 \pm 8.8^{*}$ \\
\hline \multicolumn{3}{|l|}{ Ankle joint } \\
\hline Plantar flexion [Loading response] & $1.5 \pm 3.5$ & $1.5 \pm 5.4$ \\
\hline Dorsal flexion [Terminal stance] & $20.2 \pm 4.8$ & $16.5 \pm 4.1^{* *}$ \\
\hline Plantar flexion [Pre-swing] & $-1.6 \pm 7.6$ & $6.3 \pm 7.3^{* *}$ \\
\hline Trunk anteversion [one gait cycle] & $6.9 \pm 6.1$ & $2.5 \pm 5.1^{*}$ \\
\hline \multicolumn{3}{|l|}{ Joint moment $(\mathrm{Nm} / \mathrm{kg})$} \\
\hline \multicolumn{3}{|l|}{ Hip joint } \\
\hline Extension [Loading response] & $0.56 \pm 0.23$ & $0.72 \pm 0.41$ \\
\hline Flexion [Terminal stance] & $0.34 \pm 0.28$ & $0.66 \pm 0.35 * *$ \\
\hline \multicolumn{3}{|l|}{ Knee joint } \\
\hline Flexion [Initial contact] & $0.09 \pm 0.06$ & $0.11 \pm 0.10$ \\
\hline Extension [Loading response] & $0.07 \pm 0.25$ & $0.23 \pm 0.37$ \\
\hline Flexion [Terminal stance] & $-0.09 \pm 0.30$ & $-0.07 \pm 0.33$ \\
\hline Extension [Pre-swing] & $0.13 \pm 0.14$ & $0.31 \pm 0.25 *$ \\
\hline \multicolumn{3}{|l|}{ Ankle joint } \\
\hline Dorsal flexion [Loading response] & $-0.14 \pm 0.20$ & $-0.05 \pm 0.20$ \\
\hline Plantar flexion [Terminal stance] & $0.93 \pm 0.29$ & $1.12 \pm 0.20^{*}$ \\
\hline Walking velocity $(\mathrm{m} / \mathrm{sec})$ & $0.79 \pm 0.19$ & $1.08 \pm 0.27 * *$ \\
\hline Stride length [stride length/height] $(\mathrm{m} / \mathrm{m})$ & $0.21 \pm 0.10$ & $0.29 \pm 0.09^{*}$ \\
\hline Walking rate (steps/min) & $117.83 \pm 10.96$ & $125.42 \pm 10.64^{*}$ \\
\hline
\end{tabular}

$* \mathrm{p}<0.05,{ }^{*} \mathrm{p}<0.01$.

\section{DISCUSSION}

The prevalence of sarcopenia in Japan is reported to be approximately $10-20 \%$ in community-dwelling elderly people and approximately $20-40 \%$ in frail elderly people (including facility residents) who need some support or care ${ }^{7,28-32)}$. The prevalence of sarcopenia was $47.5 \%$ in this study of outpatient rehabilitation patients or community day-care center users, 
which was similar to the prevalence in previous studies ${ }^{30-32)}$ in frail elderly people. Therefore, it is considered that the data in this study were not biased.

In the fall rate over the past year, the proportion of the sarcopenia group who experienced a fall was $31.6 \%$ while in the non-sarcopenia group it was $19.0 \%$, and the rate in the sarcopenia group tended to be higher than the non-sarcopenia group. This is considered to be due to the fact that the ability to perform activities of daily living is lower in the sarcopenia group than in the non-sarcopenia group, and the risk of falling is higher, resulting in the same results as in previous research ${ }^{7,8)}$.

With regard to lower limb and trunk muscle strength, the sarcopenia group had significantly weaker trunk extension muscle strength than the non-sarcopenia group. Therefore, it was thought that the results of this study suggested that sarcopenia may affect not only lower limb muscle but also trunk muscle in elderly people. According to a report by Ikezoe et al. ${ }^{19)}$, trunk muscles also atrophy with age. Particularly in frail elderly people who need support in walking, it has been shown that among the trunk muscles, the rate of decrease of the transversus abdominis muscle, the multifidus muscle, and the thoracic spinal erector muscle is high. In other words, it is thought that trunk muscles are also affected by sarcopenia, and it is necessary to investigate not only lower limb muscles but also trunk muscles in order to fully understand the state of sarcopenia.

When the sarcopenia group and the non-sarcopenia group were compared for the characteristics of walking, in terms of joint angles, the sarcopenia group had a significantly higher trunk anteversion angle during one gait cycle than the nonsarcopenia group. Thus it can be seen that the sarcopenia group was walking in a state of kyphosis. In general, muscle weakness in the trunk muscles can be considered as one of the causes of kyphosis. The trunk muscles maintain posture and protect the spine, so when muscle strength decreases, it is likely to cause postural changes such as kyphosis ${ }^{14,15)}$. In the sarcopenia group of this study, trunk extension muscle strength was also significantly lower than in the non-sarcopenia group. These data suggest that the sarcopenia group is likely to be in kyphosis due to weakness of the trunk muscles.

In terms of joint moments, in the sarcopenia group, the hip joint maximum flexion moment and the ankle joint maximum plantar flexion moment at the terminal stance were significantly lower than in the non-sarcopenia group. That is, the sarcopenia group was weak in swinging and kicking out of the lower limbs serving as a forward propulsive force during walking, and as a result, walking velocity was lowered. In general, muscle weakness associated with age-related muscle atrophy is considered to be one of the causes of decreased walking velocity ${ }^{33-35)}$, and in the sarcopenia group of this study, muscle strength was lower than that of general elderly people ${ }^{36}$. However, in our previous survey ${ }^{13)}$, there was no difference in lower limb muscle strength due to the presence or absence of sarcopenia, and the same results were obtained in this study. In addition, investigation of the trunk muscles revealed that the sarcopenia group was significantly weaker in trunk extension muscle strength than the non-sarcopenia group. Therefore, these data revealed that the cause of the decrease in walking velocity in elderly people with sarcopenia is not only muscle weakness of the lower limb muscles but also muscle weakness of the trunk muscles.

The limitation of this study is that it is targeted for outpatient rehabilitation patients or community day-care center users, and it is thought that there is a bias due to disease. In the future, it will be necessary to investigate the effects of diseases and investigate the characteristics of each disease. In addition, there was a gender difference when the participants were divided into two groups according to the presence or absence of sarcopenia. Therefore, it is possible that the results of this study were influenced by gender differences. In the future, it will be necessary to investigate the effects of gender.

The sarcopenia group was not significantly different in terms of lower limb muscle strength compared to the non-sarcopenia group, but was significantly weaker in trunk muscle strength. In other words, these data suggest that sarcopenia may affect not only the lower limb muscles but also the trunk muscles in elderly people. In addition, in the sarcopenia group, swinging and kicking out of the lower limbs, which are the forward driving force during walking, were weak, and a decrease in walking velocity was recognized. Therefore, it was revealed that the cause of the decrease in walking velocity in sarcopenia of elderly people is not only muscle weakness of the lower limb muscles but also muscle weakness of the trunk muscles.

\section{Conflict of interest}

None.

\section{ACKNOWLEDGEMENT}

The authors would like to thank the participants who cooperated with this research and everyone who contributed to the data collection.

\section{REFERENCES}

1) Cabinet Office: Annual report on the aging society (2019, whole version). https://www8.cao.go.jp/kourei/whitepaper/w-2019/zenbun/01pdf_index.html (Accessed Jul. 1, 2019)

2) Rosenberg I: Summary comments: epidemiological and methodological problems in determining nutritional status of older persons. Am J Clin Nutr, 1989, 50: 1231-1233. [CrossRef] 
3) Cruz-Jentoft AJ, Baeyens JP, Bauer JM, et al. European Working Group on Sarcopenia in Older People: Sarcopenia: European consensus on definition and diagnosis: report of the European Working Group on sarcopenia in older people. Age Ageing, 2010, 39: 412-423. [Medline] [CrossRef]

4) Chen LK, Liu LK, Woo J, et al.: Sarcopenia in Asia: consensus report of the Asian Working Group for sarcopenia. J Am Med Dir Assoc, 2014, 15: 95-101. [Medline] [CrossRef]

5) Baumgartner RN, Koehler KM, Gallagher D, et al.: Epidemiology of sarcopenia among the elderly in New Mexico. Am J Epidemiol, 1998, 147: 755-763. [Medline] [CrossRef]

6) Newman AB, Kupelian V, Visser M, et al. Health ABC Study Investigators: Sarcopenia: alternative definitions and associations with lower extremity function. J Am Geriatr Soc, 2003, 51: 1602-1609. [Medline] [CrossRef]

7) Yamada M, Nishiguchi S, Fukutani N, et al.: Prevalence of sarcopenia in community-dwelling Japanese older adults. J Am Med Dir Assoc, 2013, 14: 911-915. [Medline] [CrossRef]

8) Landi F, Liperoti R, Russo A, et al.: Sarcopenia as a risk factor for falls in elderly individuals: results from the ilSIRENTE study. Clin Nutr, 2012 , 31: 652-658. [Medline] [CrossRef]

9) Suzuki M, Yamada K, Takahashi H, et al.: [A study of falls among elderly living in the community]. Nihon Kango Kagakkaishi, 1993, 13: 10-19. [Medline]

10) World Health Organization: Fact sheets (16 January 2018). https://www.who.int/news-room/fact-sheets/detail/falls (Accessed Jul. 1, 2019).

11) Hayashi Y: The influence of the falls over the medical economy. Bone, 2009, 23: 181-184.

12) Shibata H: A comprehensive study on falls and fractures in community elderly. Grant-in-Aid for Scientific Research Research Result Report (1995-1996), Tokyo: Ministry of Education, 1997

13) Kato K, Hatanaka Y, Saito K: The influence of sarcopenia on walking in frail elderly people. OAJ Gerontol Geriatr Med, 2017,2 : 1-6.

14) Mika A, Unnithan VB, Mika P: Differences in thoracic kyphosis and in back muscle strength in women with bone loss due to osteoporosis. Spine, 2005, 30: 241-246. [Medline] [CrossRef]

15) Birnbaum K, Siebert CH, Hinkelmann J, et al.: Correction of kyphotic deformity before and after transection of the anterior longitudinal ligament-a cadaver study. Arch Orthop Trauma Surg, 2001, 121: 142-147. [Medline] [CrossRef]

16) Ito S: Pain and muscle strengthening: about low back pain and trunk muscle strength. Phys Ther J, 1998, 32: 847-854.

17) Toyoda H, Hoshino M, Ohyama S, et al.: The association of back muscle strength and sarcopenia-related parameters in the patients with spinal disorders. Eur Spine J, 2019, 28: 241-249. [Medline] [CrossRef]

18) Tanimoto Y, Watanabe M, Kono R, et al.: [Aging changes in muscle mass of Japanese]. Nippon Ronen Igakkai Zasshi, 2010, 47: 52-57 (in Japanese). [Medline] [CrossRef]

19) Ikezoe T, Mori N, Nakamura M, et al.: Effects of age and inactivity due to prolonged bed rest on atrophy of trunk muscles. Eur J Appl Physiol, 2012, 112: 43-48. [Medline] [CrossRef]

20) Gibson MJ, Andres RO, Isaacs B, et al.: The prevention of falls in later life. A report of the Kellogg International work group on the prevention of falls by the elderly. Dan Med Bull, 1987, 34: 1-24. [Medline]

21) Kato M, Yamasaki H, Hiiragi Y, et al.: Measurements of isometric knee extension force with a hand-held dynamometer-Effect for interrater reliability using fixing-belt. Sogo Rehabil, 2001, 29: 1047-1050.

22) Kato M, Yamasaki H, Nakajima K, et al.: Measurements of isometric hip flexion and extension force with a hand-held dynamometer-The effect of use of the belt for fixation on the reliability-. J Kochi Rehabil Inst, 2005, 6: 7-13.

23) Sugimoto S, Onozuka N: The relationship of lower extremity strength and walking ability in elderly. Sogo Rehabil, 2005, 33: 567-570.

24) Arisue I, Takeuchi $T$, Nakamoto $M$, et al.: Intra-rater reliability to measure isometric hip extension muscle strength with a hand-held dynamometer in the measurement of prone, supine, and standing positions. J Phys Ther Pract Res, 2015, 24: 13-17.

25) Souza LA, Martins JC, Teixeira-Salmela LF, et al.: Validity and reliability of the modified sphygmomanometer test to assess strength of the lower limbs and trunk muscles after stroke. J Rehabil Med, 2014, 46: 620-628. [Medline] [CrossRef]

26) Tokyo Metropolitan University Physical Fitness Standard Value Study Group: New Japanese physical fitness standard II. Tokyo: Fumaido Publishers, 2007.

27) The Clinical Gait Analysis Forum of Japan: Walk analysis by joint moment. Tokyo: Ishiyaku Publishers, 1997.

28) Yuki A, Ando F, Matsui Y, et al.: The epidemiology of sarcopenia among the Japanese elderly. J Phys Fit Sports Med, 2015, 4: 111-115. [CrossRef]

29) Taniguchi Y, Seino S, Fujiwara Y, et al.: [Cross-sectional and longitudinal associations of physical performance and skeletal muscle mass with cognition and cognitive decline among community-dwelling older Japanese]. Nippon Ronen Igakkai Zasshi, 2015, 52: 269-277 (in Japanese). [Medline] [CrossRef]

30) Kamo T, Suzuki R, Ito K, et al.: Prevalence of sarcopenia and its relation to body composition, physiological function, and nutritional status in communitydwelling frail elderly people. Jpn Phys Ther Assoc, 2013, 40: 414-420.

31) Haraguchi K, Kawaguchi M, Kimura A, et al.: Association of sarcopenia and osteoporosis in outpatients. Orthop Traumatol, 2016, 65: 588-590. [CrossRef]

32) Suzuki M, Sakai Y, Sasahara N, et al.: Dietary intake characteristics and nutritional status of hemodialysis patients with sarcopenia. J Jpn Soc Dial Ther, 2016, 49: 581-587. [CrossRef]

33) Ito H, Nagasaki H, Maruyama H, et al.: [Age related changes in the walking cycle during fastest walking in healthy male subjects]. Nippon Ronen Igakkai Zasshi, 1989, 26: 347-352 (in Japanese). [Medline] [CrossRef]

34) Kim JD, Kuno S, Soma R, et al.: Relationship between reduction of hip joint and thigh muscle and walking ability in elderly people. Jpn Soc Phys Fit Sport Med, 2000, 49: 589-596.

35) Ohsugi H, Murata S, Horie J, et al.: Examining of the factors related to gait parameters in the community dwelling elderly. Jpn J Health Promot Phys Ther, 2014, 4: 31-35. [CrossRef]

36) Hirasawa Y, Hasegawa T, Matsushita K, et al.: Isometric knee extension muscle strength in healthy subject. Phys Ther J, 2004, 38: 330-333. 